$\xi_{\mathrm{p}}$

\title{
Acute Stage of Brain Stroke Diagnosis Using Hybrid Genetic Algorithm for Optimization of Feature Selection and Classifier
}

\author{
C. Amuthadevi ${ }^{1}$, K.Meena ${ }^{2}$, K.Arthi ${ }^{3}$ \\ ${ }^{123}$ Associate Professor, Department of Computer Science and Engineering, \\ Department of Computer Science and Engineering, School of Computing, \\ Vel Tech Rangarajan Dr. Sagunthala R\&D Institute of Science and Technology, Avadi, Chennai-62, TamilNadu \\ Email: camuthadevi@gmail.com
}

\begin{abstract}
Brain Stroke is the third leading reason of death or major disabilities and needs computer guided assistance to diagnose at an earliest stage of disease. Stroke results in great physical functioning restrictions, which negatively impacts the quality of life for survivors and also care givers. MRI of brain is mainly used for accurate diagnosis even though its cost is high. In this work, a Hybrid Genetic Algorithm (HGA) is proposed for feature selection with Independent Component analysis and parameters optimization of Multi layer Perceptron (MLP). The classification results are compared with simple KNN and MLP Classifiers.
\end{abstract}

Keywords: Brain stroke diagnosis, MLP, Hybrid genetic algorithm,

\section{Introduction}

Stroke results in great physical functioning restrictions, which negatively impacts quality of life for survivors and care givers. A leading cause for serious, long-term disability is stroke. Stroke affected brain cells start dying at the rate of 12 million cells per minute thereby losing abilities controlled by the affected part. Dying cells release a chemical substance named glutamate which can affect surrounding tissues. Stroke effects range from simple arm or leg weakness to severe effects like paralysis of one side, speech loss and coma. Hence, it is necessary to identify a disease's important diagnostic features as this helps physicians diagnose quickly and correctly. Multimodal MRI identifies age and vascular territories in infarcts. Diagnosis of ischemic stroke subtype is performed in the first few days/weeks after hospitalization in most centers. To capture brain MRI, it uses a strong, uniform magnetic field. The magnetic field's strength ranges from 0.3 to 1.5 Tesla.

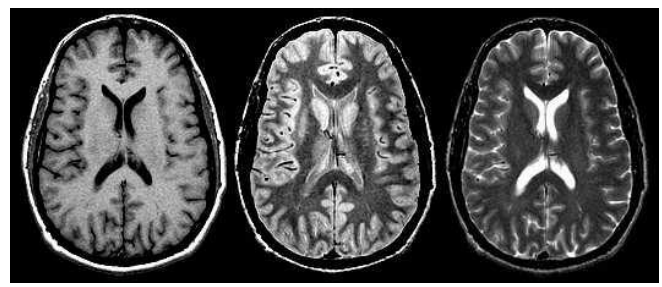

Figure 1 Sample MRI brain Images
Figure 1 shows the different types of MRI of brains. They are T1 weighted, T2 weighted and diffusion weighted MRI images. Among these modalities, DWI provides better visibility among different tissues of brains. So DWI-MRI is selected ion this work. Linear filters used for edge detection are Gabor filters. As Gabor filters frequency and orientation are similar to human visual systems, it is applied to texture representation and discrimination [1]. The features from watershed segmentation and Gabor filters are fused and then selected by ICA and hybrid GA [2]. In this work, classification by MLP is used and optimization of parameters was done by hybrid genetic algorithm.

\section{Need of Optimization}

The optimization process searches in a vector using a function producing an optimal solution. All feasible values are available solutions and the best value is the optimal solution. Optimization algorithms usually solve optimization problems. A simple way to categorize optimization algorithms is to consider the algorithm's nature. Optimization algorithms are deterministic and stochastic algorithms. Deterministic algorithms use gradients like hill climbing and hence have rigorous moves generating same set of solutions, when iterations start with same initial starting point[3] . But, stochastic algorithms do not use gradients and generate different solutions even with same initial value. But, the final values, though different, converge to same optimal solutions accurately. Stochastic algorithms are of heuristic and metaheuristic types. Heuristic algorithms are dependent on the nature of the 
problems. Most of these algorithms use greedy method. But metaheuristic algorithms are problem independent and used in many of the machine learning algorithms.

Nature-inspired metaheuristic algorithms performed powerfully / efficiently to solve modern, nonlinear numerical global optimization problems. All metaheuristic algorithms to some extent strive to balance randomization (global search) and Local Search (LS). Hybrid optimizations are a new class of optimization heuristics where two or more algorithms are used for same optimization to ensure best results. A hybrid optimization uses a heuristic to select the best algorithm to apply in a situation. A hybrid optimization reduces compilation effort, using an efficient algorithm but occasionally uses a better effective/expensive optimization algorithm when the additional benefit is worth it.

Most meta-heuristic feature subset selection algorithms use wrapper approach due to inherent advantages [4]. In filter approach, feature selection is a pre-processing step and its disadvantage is that it ignores effect of chosen feature subset on the induction algorithm's performance. It is claimed[4] that to determine a useful features subset, the subset selection algorithm must consider induction algorithm's biases to choose a subset. This work builds a wrapper approach model using ANN as classification function.

\section{Related Works}

Othman \& Basri [5] employed and implemented the Probabilistic Neural Network (PNN) with image and data processing techniques for an automated brain tumor classification. MRIs containing noise caused by the operator leading to serious errors during the classification. The use of NN and fuzzy logic is observed to have great potential to improve the classification. The performance of the PNN classifier was evaluated in terms of training performance and classification accuracies. PNN gave fast and accurate classification and was a promising tool for classification of the tumors.

Sridhar \& Murali Krishna [6] proposed a novel technique for Brain Tumor Classification using PNN with DCT. The proposed framework extracted the features using DCT and applied dimensionality reduction techniques and then the classification was carried out using PNN. To evaluate the proposed technique, an image data base of 20 Brain Tumor images was used. The proposed method gave fast and better recognition rate when compared with conventional classifiers. The main advantage of this method was its high speed processing capability and low computational requirements.

Larsen et al [7] investigated about the perfusion measured with Dynamic Contrast-Enhanced Magnetic Resonance Imaging (DCEMRI) for differentiating radiation necrosis from tumor recurrence in patients with high-grade glioma. The method was adept in classifying fourteen enhancing lesions as progressing or regressing. An empirical threshold of $2.0 \mathrm{ml} / 100 \mathrm{~g}$ for CBV allowed detection of regressing lesions with a sensitivity of $100 \%$ and specificity of $100 \%$. Fluro Denoxy Glucose -PET (FDG-PET) and DCE-MRI agreed in classification of tumor status in 13 out of the 16 cases where an FDG-PET classification was obtained.

Saritha et al [8] proposed a new approach by integrating wavelet entropy based spider web plots and PNN for the classification of
MRI brain images. The spider web plot was a geometric construction drawn using the entropy of the wavelet approximation components and the areas calculated were used as feature set for classification. PNN provided a general solution to the pattern classification problems and the classification accuracy was found to be $100 \%$.

Paul \& Bandhyopadhyay [9] showed that the success rate of the segmentation in images of brain MRI taken from all the three angles was quite high and satisfactory. The real time execution time in the test cases was less than 9 seconds in almost all the cases and thus could be said to be good as per the current industry standard and was also very less as compared with manual process. Similar results were also available in axial view MRI Images. Experiments conducted with nearly hundred MRI images demonstrated successful tumor detection in more than $96 \%$ cases.

Amin \& Megeed [10] analyzed different segmentation and feature extraction combinations with MLP classifier. The authors showed that the PCA / MLP had a peak recognition rate of $100 \%$ and average recognition rate of $78.2 \%$. Segmentation / MLP results had a peak recognition rate of $96.7 \%$ and average recognition of $78 \%$. Experimental results also showed that the time taken to classify the segmented images was much less than that was taken to classify the feature vector resulting from applying PCA network.

Dahab et al [11] presented a modified version of the conventional PNN method that could successfully handle the process of MRI image classification with $100 \%$ accuracy when the spread value was equal to 1 . It was also concluded that the proposed Learning Vector Quantization (LVQ)-based PNN system decreased the processing time to approximately $79 \%$ compared with the conventional PNN and despite considerable progress in PNN, there had been a room for improvement as far as network structure determination was concerned. Kumari \& Mehra [12] presented the classification accuracy of DWT+PCA+ANN method was improved by $95.7 \%$ when compared with DWT+Self Organizing Map (SOM) classifier, HAAR+PSO+SVM, and KNN classifier methods.

John [13] evaluated the percentage of accuracy of classification using PNN and it was found to be nearly $100 \%$, when the spread value was set to 1 . Based on this result PNN was considered to have major advantages over conventional $\mathrm{NN}$, due to the fact that PNN learns from the training data instantaneously. This speed of learning made the PNN to of adapt in real time learning. This method of automatic early detection and classification of MRI brain into normal, benign and malignant, based on their statistical texture features, not only replaces conventional invasive techniques, but also helps in reducing the fatality rate. Using a classifiers ensemble to decode visual stimuli from functional Magnetic Resonance Imaging (fMRI) data was proposed by Cabral et al[14]. Each classifier in ensemble specialized in a stimulus using an optimized feature set for that specific stimulus. Output for every individual stimulus was got from corresponding classifier and final classification achieved by choosing the best score. The new method was applied to two empirical fMRI datasets from multiple subjects performing visual tasks with 4 classes of stimuli. Results showed that a classifiers ensemble must provide an advantageous alternative to single classifiers when decoding stimuli linked to specific brain areas.

Neonatal brain segmentation algorithms in literature focused on by Devi et al [15] ensured an overview of clinical MRI of a new born brain and challenges in neonatal brain MRI automated tissue 
classification. It presented a detailed survey of recent segmentation-methods and their salient features. Different approaches were categorized into intracranial and brain tissue segmentation algorithms based on their tissue classification level. Also, brain tissue segmentation techniques based on their atlas usage were grouped into atlas-based, augmented atlas-based and atlas-free methods. Additionally, research gaps and lacunae in literature were also identified.

Kharat et al [16] presented two NN techniques for the classification of the human brain MRIs. The proposed NN technique consisted of three stages, namely feature extraction, dimensionality reduction, and classification. ANNs were developed for a wide range of applications such as function approximation, feature extraction, optimization, and classification. In particular, they were developed for image enhancement, segmentation, registration, feature extraction, and object recognition and classification. Among these, object recognition and image classification was more important as it was a critical step for high-level processing such as brain tumor classification. Multi-Layer Perceptron (MLP), Radial Basis Function (RBF), Hopfield, Cellular, and Pulse-Coupled neural networks were used for image segmentation. These networks could be categorized into feed-forward (associative) and feedback (auto-associative) networks.

Selvanayaki [17] presented the intelligent system that showed that the Particle Swarm Optimization (PSO) was an extremely simple and accurate algorithm for brain tumor detection. PSO gives 99.28\% of accurate detection than Ant Colony Optimization (ACO) and GA. So it seems to be effective for optimizing a wide range of functions. In past several years, PSO was successfully applied in many research and application areas. Another reason that PSO was attractive is that there are few parameters to adjust.

A novel hybrid machine learning system based on GA and SVM for brain tumor classification was proposed by Sachdeva et al [18]. Input was tumors texture/intensity features. GA selected a set of most informative input features. The study was performed on 55 patients real 428 post contrast T1-weighted MR images. Results showed that GA optimization technique enhanced overall SVM accuracy from $56.3 \%$ to $91.7 \%$. It also revealed that GA-SVM ensured more accurate results than earlier methods and was tested on diversified datasets.

\section{Methodology}

This section describes a Hybrid GA to optimize feature selection and MLP NN parameter optimization. The work proposes a new hybrid GA based algorithm for feature selection and to optimize MLP classifier to label input brain DWI image as Stroke or NonStroke. Features are obtained from a Region of Interest (ROI) using Independent Component Analysis (ICA). It is proposed to use hybrid GA to select best features set for classification. MLP classifier is trained using selected features to label input images.

Learning rate and momentum in MLP are two parameters related to convergence speed and having an important role in classification accuracy. Learning rate controls weights changes, when error value is propagated from output layer neurons to input layer neurons through hidden layers. Hence it affects training speed. Values range from 0.0 to 1.0. If very large value is chosen, classification accuracy reduces and training is very fast. If very low value is chosen, computational effort is wasted with no classification accuracy improvement. Hence, an ideal value must be chosen.

Momentum indicates learned weighs influence in an earlier iteration to current iteration. Improper value selection results in divergence. So, class differentiation does not occur and trained MLP results in a single class for all input vectors.

Table 1 shows the parameters used in this MLP NN and optimization for tuning parameters of MLP.

Table 1: MLP and GA parameters

Architecture of MLP

Number of neurons in input Number of features selected by GA

layer

Number of neurons in hidden Half of the number of neurons in input layer layer

Number of neurons in output 2

layer

\begin{tabular}{l|l}
\hline Learning rate & 0.1
\end{tabular}

\begin{tabular}{|l|l}
\hline Momentum & 0.2 \\
\hline
\end{tabular}

\begin{tabular}{|l|l|}
\hline Activation Function & Sigmoidal function in both hidden and
\end{tabular}

\begin{tabular}{|l|l}
\hline & $\begin{array}{l}\text { Sigmoidal fur } \\
\text { output layer }\end{array}$ \\
\hline
\end{tabular}

\begin{tabular}{|l|l|}
\hline Number of epochs & 500 \\
\hline
\end{tabular}

GA parameters

\begin{tabular}{|l|l|}
\hline Initial Population & 20 \\
\hline
\end{tabular}

\begin{tabular}{|l|l|}
\hline Encoding & Real Encoding \\
\hline
\end{tabular}

\begin{tabular}{|l|l|}
\hline Selection Mechanism & Roulette Wheel \\
\hline
\end{tabular}

\begin{tabular}{|l|l}
\hline Cross over & Two point cross over \\
\hline
\end{tabular}

\begin{tabular}{|l|l|}
\hline Cross over rate & 0.9
\end{tabular}

\begin{tabular}{l|l} 
Mutation rate & 0.001
\end{tabular}

\begin{tabular}{|l|l|}
\hline Minimum value of learning & 0.001
\end{tabular}

rate

\begin{tabular}{|l|l}
\hline Maximum value of learning & 0.8
\end{tabular}

\begin{tabular}{ll|l} 
Minimum value of momentum & 0.1
\end{tabular}

\begin{tabular}{l|l}
\hline Maximum value of & 0.98
\end{tabular}

momentum

MLP parameters are optimized by combining GA and Local Search (LS). The proposed MLP NN - HGA optimization performance is evaluated with, precision, recall and classification accuracy.

\section{Hybrid Genetic Algorithm}

Hybrid optimization is applied to choose best one among GA created solutions. Local Search (LS) is a meta-heuristic technique to solve hard optimization problems. LS algorithms move from a solution to another applying local changes till an optimal solution occurs before time lapses. LS is an iterative algorithm that moves from a solution $\mathrm{S}$ to another $\mathrm{S}$ ' based on a neighborhood structure. LS procedure is mentioned as follows:

Step i) Initialization: An initial schedule $\mathrm{S}$ is chosen and considered current solution to compute objective function $F(S)$ value.

Step ii) Neighbor Generation: Neighbor S' of current solution $S$ is chosen and $\mathrm{F}\left(\mathrm{S}^{\prime}\right)$ computed.

Step iii) Acceptance Test: Testing identifies whether move is accepted from $S$ to $S$ '. If accepted, then $S^{\prime}$ replaces $S$ as current solution; otherwise $S$ is retained. 
Step iv) Termination Test: This reveals whether the algorithm has to be terminated. If it terminates, output best solution generated; or else return to neighbor generation step.

Step i explains a starting solution obtained or it can be a random feature permutation. If a LS procedure is applied many times, then it is reasonable to use random initial schedules. To generate a neighbor S' in Step ii, a neighborhood structure has to be specified.

Various neighborhood types are,

-Transpose neighborhood occupies two values that are adjacent in the sequence and can be interchanged. For example $(1,2,3,4,5,6,7) \circledast(1,3,2,4,5,6,7)$,

-Swap neighborhood interchanges two arbitrary values like $(1,2,3,4,5,6,7) \circledast(1,6,3,4,5,2,7)$,

-Insert neighborhood removes one value from current position and inserts it elsewhere like $(1,2,3,4,5,6,7)$ (B) $(1,3,4,5,6,2,7)$. Neighbors are generated randomly, systematically, or by a combination of both approaches.

In this work, swap neighborhood method is used. The number of iterations was fixed as 500. The flow chart of hybrid optimization used in this work is given in the Figure 2.

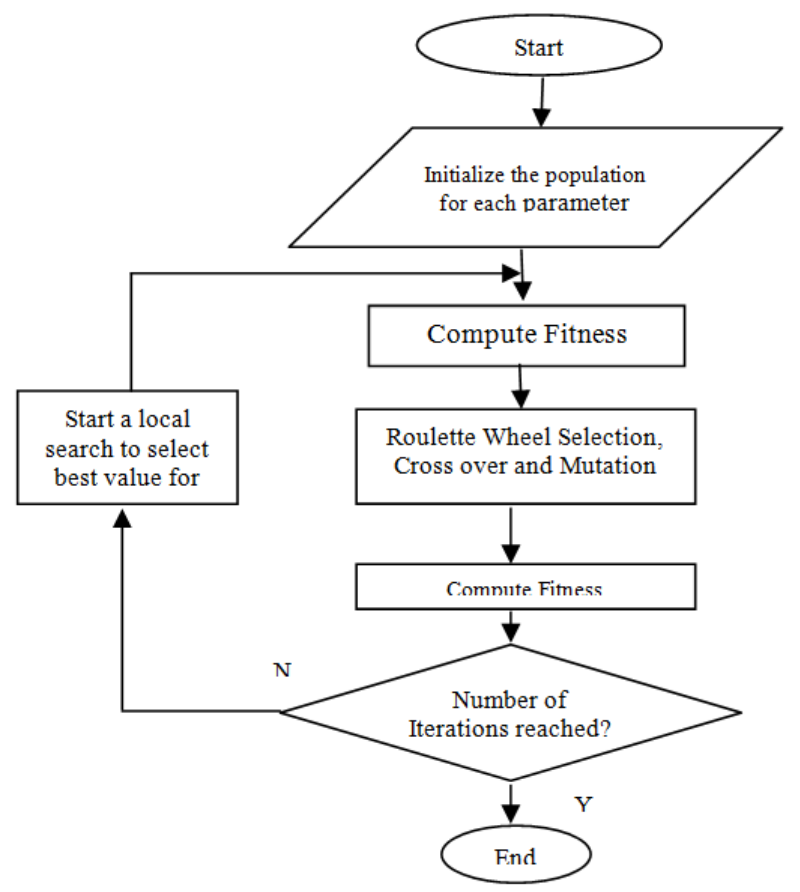

Figure 2 Flow chart of the hybrid optimization

Hybrid GA-LS optimizes MLP weights. Chromosomes are encoded using real encoding where a gene represents MLP weights. Initial population is randomly chosen. The population's fitness is evaluated and iterated through the algorithm till termination. A new fitness function is designed with correlation and MSE. Equation (1) gives the new fitness function $\mathrm{f}$.

$f=\alpha \beta(1-\alpha)(1-\beta) M S E$
The values of $\alpha, \beta$, and $\gamma$ are calculated by the equations,

$\alpha=\frac{T P+F N}{N}$

$\beta=\frac{T P+F P}{N}$

where, TP - number of True positives, FP - number of false positives and and FN - number of false negatives achieved in image classification. Here, $\mathrm{N}$ is number of classification instances.

\section{Results and Discussion}

For the experiments, 80 stroke and 220 normal MRI images are used. The performance is evaluated for: Features are extracted from watershed segmentation algorithm and gabor filter and fused with normalized concatenation.

- $\quad$ FUHGAR - Fused feature extraction with Hybrid GA based feature selection \& RMSE objective

- $\quad$ FUHGAP - Fused feature extraction with Hybrid GA based feature selection \& Proposed objective

Table 3 gives the classification accuracy and RMSE of HMLP classifier.

Table 3 Classification Accuracy for Proposed hybrid method

\begin{tabular}{|l|l|c|}
\hline & \multicolumn{1}{|c|}{ Classification Accuracy } & RMSE \\
\hline & KNN classifier & 0.1341 \\
\hline FUHGAR & 94.67 & 0.1318 \\
\hline FUHGAP & 95 & \\
\hline & MLP NN classifier & \\
\hline FUHGAR & 96.63 & 0.1234 \\
\hline FUHGAP & 96.67 & 0.1224 \\
\hline & MLP NN with HGA-optimization & \\
\hline FUHGAR & 97.33 & 0.1098 \\
\hline FUHGAP & 98.33 & 0.0834 \\
\hline
\end{tabular}

The classification accuracy of the classifiers is shown as graphically in Figure 3. In Figure 4, the best fitness values against the number of iterations are plotted for the several classifiers.

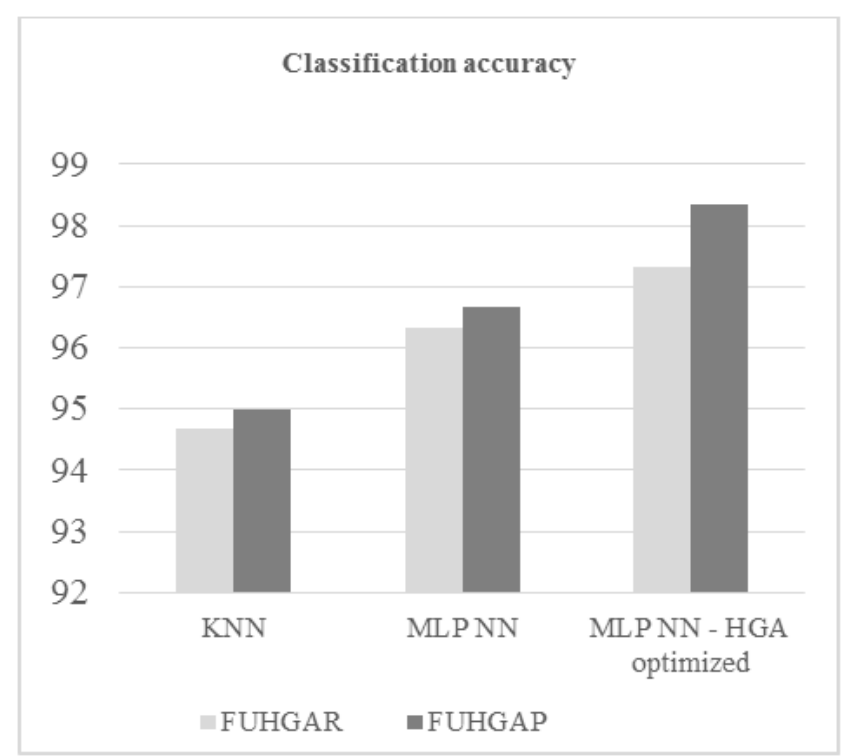

Figure 3 Classification Accuracy 
Figure 3 shows the comparison of classification accuracy with different classifiers. Result graph showed that the accuracy of the proposed MLP NN - HGA optimized with fused feature extraction with Hybrid GA based feature selection \& proposed objective increased by $1.7 \%$ compared with MLP NN with fused feature extraction with Hybrid GA based feature selection \& proposed objective.

Table 4 lists the precision, recall, and $\mathrm{f}$ measure of HMLP classifier for normal and stroke images.

Table 4 Precision, Recall, and F measure

\begin{tabular}{|c|c|c|c|}
\hline & KNN & MLP NN & \begin{tabular}{c|} 
MLP NN - \\
HGA optimized
\end{tabular} \\
\hline \multicolumn{4}{|c|}{ Precision for Stroke } \\
\hline FUHGAR & 0.9103 & 0.9367 & 0.9737 \\
\hline FUHGAP & 0.9221 & 0.9605 & 0.9747 \\
\hline \multicolumn{4}{|c|}{ Precision for Normal } \\
\hline FUHGAR & 0.9595 & 0.9729 & 0.9732 \\
\hline FUHGAP & 0.9596 & 0.9688 & 0.9864 \\
\hline \multicolumn{4}{|c|}{ Recall for stroke } \\
\hline FUHGAR & 0.8875 & 0.925 & 0.925 \\
\hline FUHGAP & 0.8875 & 0.9125 & 0.9625 \\
\hline \multicolumn{4}{|c|}{ Recall for Normal } \\
\hline FUHGAR & 0.9682 & 0.9773 & 0.9909 \\
\hline FUHGAP & 0.9727 & 0.9864 & 0.9909 \\
\hline \multicolumn{4}{|c|}{ F measure for stroke } \\
\hline FUHGAR & 0.8988 & 0.9308 & 0.9487 \\
\hline FUHGAP & 0.9045 & 0.9359 & 0.9686 \\
\hline \multicolumn{4}{|c|}{ F measure for Normal } \\
\hline FUHGAR & 0.9638 & 0.9751 & 0.982 \\
\hline FUHGAP & 0.9661 & 0.9775 & 0.9886 \\
\hline
\end{tabular}

From Table 4, it can be observed that for stroke, the proposed MLP NN - HGA optimized with fused feature extraction with Hybrid GA based feature selection \& proposed objective increased the precision by $1.47 \%$ compared with MLP classifier with fused feature extraction with Hybrid GA based feature selection \& proposed objective. The proposed MLP NN - HGA optimized with fused feature extraction with Hybrid GA based feature selection \& proposed objective increased the recall by $5.37 \%$ compared with MLP classifier with fused feature extraction with Hybrid GA based feature selection \& proposed objective for stroke. The proposed MLP NN - HGA optimized with fused feature extraction with Hybrid GA based feature selection \& proposed objective increased the f measure by $3.44 \%$ compared with MLP classifier with fused feature extraction with Hybrid GA based feature selection \& proposed objective for stroke.

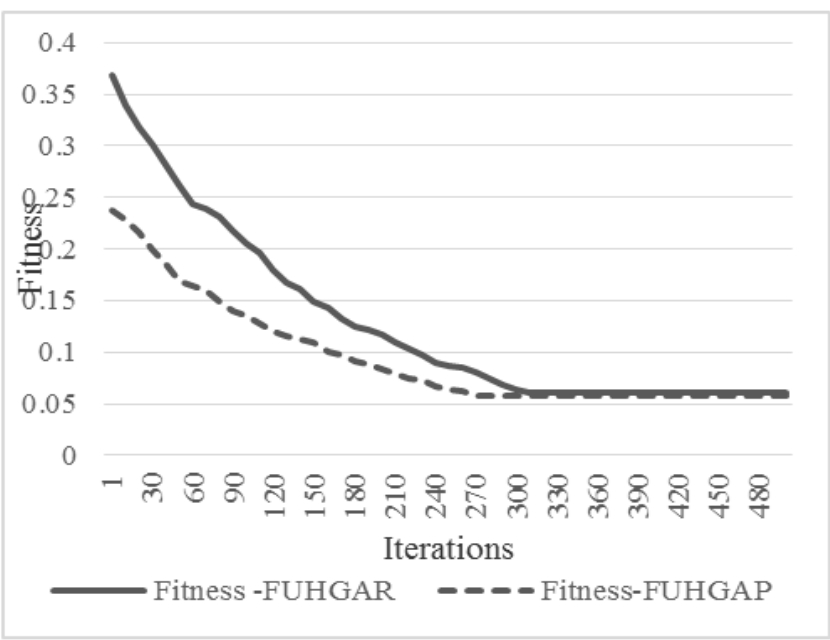

Figure 5 Best Fitness

From the Figure 5, it is observed that the proposed classifier converges at earlier iterations than simple RMSE objective.

\section{Conclusion}

An ANN based classifier was trained to classify DWI images as stroke/non-stroke images in this work. The current investigation aimed to verify the new optimized feature selection performance based on ICA and the new Hybrid GA optimized MLP NN classifier to classify stroke and non-stroke images. Hybrid GA algorithm selected the best features set for ANN classifier. ANN's learning parameters were tuned by hybrid optimization. Numerical results revealed that the proposed classifier's precision, recall and classification improved compared to simple MLP and GA-MLP classifiers with $\mathrm{NN}$ extracted features.

\section{References}

[1] AmuthaDevi, C \& Rajagopalan, SP 2013, 'Brain Stroke Classification Based On Multi-Layer Perceptron Using Watershed Segmentation And Gabor Filter', Journal of Theoretical and Applied Information Technology, vol.56, no. 2, pp. 410-416.

[2] Amutha Devi, C \& Rajagopalan, SP 2015, 'Independent Component Analysis With Genetic Algorithm Feature Selection for Ischemic Stroke Classification', Journal of Pure and Applied Microbiology, vol. 9, Special Issue On Recent Research Challenges in Bio-Medical Applications, pp. 215-226

[3] Wang, G \& Guo, L 2013, 'A novel hybrid bat algorithm with harmony search for global numerical optimization', Journal of Applied Mathematics, available from <http://www.hindawi.com/journals/jam/2013/696491/cta/>.

[4] Boz, O, 2002, 'Feature Subset Selection by Using Sorted Feature Relevance, In ICMLA,pp.147-153.

[5] John, GH, Kohavi, R \& Pfleger, K 1994, 'Irrelevant features and the subset selection problem', In the proceedings of the Eleventh International Conference in Machine Learning, pp. 121-129.

[6] Othman, MF \& Basri, MAM 2011, 'Probabilistic neural network for brain tumor classification', In IEEE Second International Conference on Intelligent Systems, Modelling and Simulation (ISMS), pp. 136-138.

[7] Sridhar, D \& Murali Krishna, IV 2013, 'Brain tumor classification using discrete cosine transform and probabilistic neural network', In IEEE International Conference on Signal Processing, Image Processing \& Pattern Recognition (ICSIPR), pp. 92-96. 
[8] Saritha, M, Joseph, KP \& Mathew, AT 2013, 'Classification of MRI brain images using combined wavelet entropy based spider web plots and probabilistic neural network', Pattern Recognition Letters, vol. 34, no. 16, pp. 2151-2156.

[9] Paul, TU \& Bandhyopadhyay, SK 2012, 'Segmentation of Brain Tumor from Brain MRI Images Reintroducing K-Means with advanced Dual Localization Method', International Journal of Engineering Research and Applications (IJERA), vol. 2, no 3, pp. 226-231.

[10] Amin, SE \& Megeed, MA 2012, 'Brain tumor diagnosis systems based on artificial neural networks and segmentation using MRI', In IEEE 8th International Conference on Informatics and Systems (INFOS), pp. MM-119.

[11] Dahab, DA, Ghoniemy, SS \& Selim, GM 2012, 'Automated Brain Tumor Detection and Identification Using Image Processing and Probabilistic Neural Network Techniques', International journal of image processing and visual communication, vol. 1 , no. 2 , pp. 1-8

[12] Kumari, A \& Mehra, R 2014, 'Design of hybrid method PSO and SVM for detection of brain neoplasm', International Journal of Engineering and Advanced Technology, vol. 3, no. 4, pp. 262266

[13] John, P 2012, 'Brain tumor classification using wavelet and texture based neural network', International Journal of Science, Engineering Research, vol. 3, no. 10, pp. 1-7.

[14] Cabral, C, Silveira, M \& Figueiredo, P 2011, 'Decoding visual stimuli using classifier ensembles with optimized feature selection', In IEEE International Symposium on Biomedical Imaging: From Nano to Macro, pp. 300-304.

[15] Devi, CN, Chandrasekharan, A, Sundararaman, VK \& Alex, ZC 2015, 'Neonatal brain MRI segmentation: A review', Computers in biology and medicine, vol. 64, pp. 163-178.

[16] Kharat, KD, Kulkarni, PP \& Nagori, MB 2012, 'Brain tumor classification using neural network based methods', International Journal of Computer Science and Informatics, vol. 1, no .4, pp.85-90.

[17] Selvanayaki, K 2013, 'Intelligent brain tumor tissue segmentation from magnetic resonance image (MRI) using meta heuristic algorithms', Journal of Global Research in Computer Science, vol. 4, no. 2, pp. 13-20.

[18] Sachdeva, J, Kumar, V, Gupta, I, Khandelwal, N \& Ahuja, CK 2011, December, 'Multiclass brain tumor classification using GA-SVM', In the proceedings of IEEE conference on Developments in E-systems Engineering (DeSE), pp. 182-187. 